\title{
A Density-based Energy-efficient Clustering Heterogeneous Algorithm for Wireless Sensor Networks
}

\author{
Zhanyang $\mathrm{Xu}^{1,2}$, Yue Yin ${ }^{1,2}$, Jin Wang ${ }^{1,2}$ and Jeong-Uk Kim ${ }^{3}$ \\ ${ }^{1}$ School of Computer and Software, Nanjing University of Information Science \& \\ Technology, Nanjing 210044, China, ${ }^{2}$ Jiangsu Engineering Center of Network \\ Monitoring, Nanjing University of Information Science \& Technology, 210044, Chin, \\ ${ }^{3}$ Department of Energy Grid, Sangmyung University, Seoul 110-743, Korea
}

\begin{abstract}
Clustering is an efficient method adopted in various routing algorithms for wireless sensor networks. However, most clustering algorithms are not suitable for heterogeneous networks. In this paper, we propose a Density-based Energy-efficient Clustering Heterogeneous Algorithm (DECHA). In DECHA, we define the density of a node and together with its energy condition to adjust the probability for the candidate cluster head selection dynamically. Candidate cluster heads further evaluate the energy level of its neighbors and adjust to find more proper cluster heads. Moreover, we design an intra-cluster algorithm as well as a multi-hop inter-cluster routing algorithm. Simulation results show that cluster heads are properly deployed in a heterogeneous wireless sensor network. Compared with some popular algorithms, in our DECHA, the stability period and network lifetime and prolonged and total energy consumption is prominently reduced.
\end{abstract}

Keywords: wireless sensor networks; heterogeneous; clustering; density; muti-hop

\section{Introduction}

The wireless sensor network (WSN) [1] is currently a research hot spot both at home and aboard. WSN consist of a great number of sensor nodes that work cooperatively to implement real-time information collection and monitoring of the target field. It combines sensor technology, information technology and network communication technology. Sensor nodes collect data and pass to the base station. WSNs have been applied in many fields such as military, agriculture and health care etc. and have broad application prospects in the future.

Routing in WSNs has been the subject of intense research efforts for years. The essence of routing algorithms is to find an optimal path that enables the efficient exchange of information between source nodes and base station, and to ensure correct transmission of data along the path. As the battery, capability of computing, storage and data processing of a sensor are limited, the energy efficiency of the network has been one of the top concerns of designing WSN routing algorithm.

Clustering is widely adopted in WSNs, where the entire network is divided into multiple clusters. Each cluster has one cluster head $(\mathrm{CH})$ and it is responsible for data aggregation. Instead of direct communication with the sink, all the member nodes in one cluster send data to the $\mathrm{CH}$. In this way, the traffic load can be reduced. It has the advantages of low energy consumption, simple routing scheme and good scalability, and it reduce the energy hole problem to some extent.

Traditional clustering routing protocols for WSN are mostly based on homogeneous networks where all sensor nodes are identical in terms of battery energy and hardware 
configuration. It is evident that cluster heads turn to be overloaded with the long range transmission cost for delivering data to the base station and the extra processing cost for data aggregation and coordination. In result, cluster heads expire before other normal nodes. Due to the variation of nodes' resources and possible topology change of the network, heterogeneous senor networks [2] are more practical in reality. In such networks, two or more types of nodes with different battery energy and processing capability are deployed. The presence of heterogeneous nodes with enhanced capacity in a sensor network is known to increase network reliability and lifetime [3].

Moreover, with intra-cluster multi-hop transmission into consideration, cluster heads that are closer to the sink often have to relay data for others besides sending data of its own interest, so that their energy consumption rate is much higher than the remote ones. They are under risk of running out of energy and becoming invalid. To further attenuate such energy hole problem, it is possible to rotate the role of $\mathrm{CHs}$ randomly and periodically change as proposed in LEACH [4].

In this paper, we have some senor nodes equipped with additional energy resources than other normal nodes. We have assumed that all sensor nodes are uniformly distributed. We propose a Density-based Energy-efficient Clustering Heterogeneous Algorithm (DECHA). Following the thoughts of LEACH, it is based on weighted election probabilities of each node to become cluster heads. In DECHA, we consider the position information of nodes, define the density of each node as the number of its neighbor nodes, and together with the energy capacity, regard it as an important evaluation metric for electing candidate CHs. DECHA also sets further adjustments to seek more proper $\mathrm{CHs}$, thus promote both lifetime and energyefficiency. An intra-cluster algorithm and a multi-hop inter-cluster routing algorithm are designed, which does save energy.

The rest of the paper is organized as follows. Section 2 introduces some related work. In Section 3, the algorithm is described in details. We set relevant models, discuss the optimal probability, elaborate the procedure of $\mathrm{CH}$ selection and set forth intra-cluster and inter-cluster routing algorithms. Simulation evaluation and performance comparison are given in Section 4 and Section 5 concludes this paper.

\section{Related work}

Low Energy Adaptive Clustering Hierarchy (LEACH) [4] is a classical clustering algorithm in homogeneous WSNs. In a periodical way, it randomly chooses the cluster heads. Each node has a probability of $\mathrm{P}$ performed as a determined prior to become one cluster head, as is shown in Eq. (1).

$$
T(n)=\left\{\begin{array}{ll}
\frac{P}{1-P[r \bmod (1 / P)]} & \text { if } n \in G, \\
0 & \text { otherwise }
\end{array}\right\}
$$

where $T(n)$ represents the threshold value of node $n, r$ is the current round index, and $G$ is a set of unselected cluster heads of the nodes in the previous rounds. Nodes are evaluated on whether or not to be cluster heads first and the un-chosen nodes join to the nearest clusters.

In LEACH, the energy consumption of entire network is evenly distributed to each sensor node, which aims to reduce energy consumption and improve the network lifetime. The algorithm is simple, however, it has some deficiencies: First, it does not guarantee about even distribution of cluster heads over the network. Some very big clusters and very small clusters may exist in the network at the same time. Second, cluster head selection is unreasonable in heterogeneous networks where nodes have 
different energy. Third, in this protocol it is assumed that each cluster head transmits data to base station over a single hop, which may consume much energy.

Besides LEACH, various clustering algorithms have been proposed for homogeneous WSNs. In Ref. [5], each node communicates only with a close neighbor and takes turns transmitting to the base station. Ref. [6] elects cluster heads based on the average minimum reachability power. Ref. [7] considers the tradeoff of the energy expenditure between nodes to cluster heads and cluster heads to base station. Global knowledge of distance is required though. Such algorithm suffers from the energy hole problem. Ref. [8] adopts fuzzy logic to overcome the defects of LEACH. It studies that using fuzzy variables can prolong the network lifetime in homogeneous network system.

Election of cluster heads plays a significant role. In many researches, nodes' position and connectivity have been focused. In Ref. [9] is applied as a node-weight heuristic algorithm with node's residual energy, number of nodes in the neighbor partition and relative location under consideration. Ref. [10] proposes a cluster allocation and routing algorithm based on node density and study on optimal density proportion for deploying sensor nodes. In Ref. [11], the cluster-head selection depends on remaining energy level of sensor nodes for transmission. Ref. [12] forms a cluster network with required coverage and connectivity and it avoids collisions and overhearing of data packets. Ref. [13] is an improvement for LEACH on the basis of nodes' connectivity. A metric of nodes' relative density is introduced for cluster-head selection. It set nodes in dense area to have larger probability to become a cluster head.

Examples of popular heterogeneous algorithms are introduced. SEP [14] is a heterogeneous-aware protocol that sets two types of nodes according to the initial energy. It aims to prolong the time interval before the death of the first node, which is referred to as the stability period. However, it is not fit for the widely used multi-level heterogeneous wireless sensor networks, which include more than two types of nodes. In DEEC [15], the cluster-heads are elected by a probability based on the ratio between the residual energy of each node and the average energy of the network. DEEC adapt the rotating epoch of each node to its energy. The nodes with high initial and residual energy will have more chances to be the cluster-heads than the low-energy nodes. Simulations show that DEEC can achieve longer network lifetime However, both SEP and DEEC fail to consider the location of the elected cluster heads in each round. It is possible that cluster heads are so close that remote nodes suffer from long range transmission cost. According to our research, further improvement is proposed and it can be adopted in multi-level heterogeneous wireless sensor networks.

There are other routing algorithms for heterogeneous wireless sensor networks. Ref. [16] has cluster heads respectively perform data fusion and data communication. Nodes with higher residual energy, lower communication cost and more strong data processing capacity, will be prior to become the cluster-head. In Ref. [17], weighted election probabilities of each node to become a cluster head are set according to the residual energy in each node. Ref. [18] introduces a weighted factor which can be adjusted to optimize the election probability of an advanced node or a normal one to be a cluster head. Relationship of heterogeneous parameters is discussed. Ref. [19] studies on a three-level heterogeneous network. Considers the occasion that all normal, advance and super nodes have same probability for $\mathrm{CH}$ selection and define the absolute residual energy level to avoid unnecessary punishment. Ref. [20] is dynamic and depends on local (inter-cluster) information of about energy remaining in sensor nodes without requirement of global knowledge of residual energy of the network. 


\section{Our proposed DECHA Algorithm}

\subsection{Relevant models}

We consider the scenario that the network is composed of $N$ sensor nodes, denoted as: $\left\{s_{1}, s_{2}, \ldots, s_{N}\right\}$ respectively. They are randomly deployed in a $M \times M$ square region. The nodes always have data to transmit to a base station, denoted as $B S$, which is assumed to be at the center of the area. They continuously monitor the surrounding environment. We make the following assumptions:

1) Sensor nodes are heterogeneous as some are equipped with relative larger energy than others. They are stationary after deployment.

2) Nodes can adjust their transmission power according to the relative distance to receiver

3) Links are symmetric. A node can compute the approximate distance to another node based on the received signal strength, once the transmitting power is given.

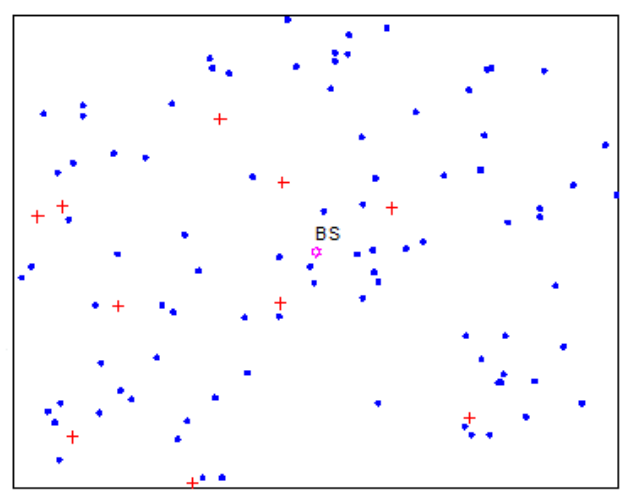

Figure 1. Network model

Figure 1 shows an example of the network model. We have " $\sqcup$ " represent the normal nodes while "+" stands for advance nodes with relative larger energy capacity.

We use similar energy model as Ref. [21]. Each sensor node will consume the following $E_{T x}$ amount of energy to transmit a $l$-bits packet over distance $d$, where the $E_{\text {elec }}$ is the energy dissipated per bit to run the transmitter or receiver circuit, $\varepsilon_{f s}$ and $\varepsilon_{m p}$ represent the transmitter amplifier's efficiency and channel conditions:

$$
E_{T x}(l, d)=\left\{\begin{array}{l}
I E_{\text {elec }}+l \varepsilon_{s f} d^{2}, d<d_{o} \\
I E_{\text {elec }}+\varepsilon_{\text {mp }} d^{4}, d \geq d_{o}
\end{array}\right.
$$

To receive a packet, radio consumes energy

$$
E_{R x}(l)=l E_{\text {elec }}
$$

Cluster heads aggregate $n$ 1-bits packets received from its members into a single 1-bits fixed packet. The energy consumption is calculated as, where $E_{D A}$ is the data aggregation cost of a bit per signal:

$$
E_{\text {aggregation }}(n, l)=n l E_{D A}
$$




\subsection{Optimal number of clusters}

For certain network, if the cluster number is too small, many sensor nodes have to send data to the base station, which consumes much energy; On the contrary, if the cluster number is too large, thus clustering becomes unnecessary. Therefore, we try to find a relative optimal cluster number $k$. For simplicity, we assume all energy calculations follow the free space channel model. With $d_{t o C H}$ representing the distance between the member node and its cluster head, the energy consumption is equal to:

$$
E_{\text {member }}=l E_{\text {elec }}+l \varepsilon_{\text {fs }} d_{\text {toCH }}^{2}
$$

Energy dissipated in the cluster head during a round includes the energy consumption of data reception, aggregation and transmission. It is given by Eq. (6) where $d_{t o B S}$ represents its distance to the base station.

$$
E_{C H}=\left(\frac{N}{k}-1\right) l E_{\text {elec }}+l E_{D A}+\left(l E_{\text {elec }}+l \varepsilon_{f s} d_{t o B S}^{2}\right)
$$

Total energy consumption of the entire network is:

$$
\begin{aligned}
& E_{\text {total }}=k\left(E_{C H}+\left(\frac{N}{k}-1\right) E_{\text {member }} \approx E_{C H}+\frac{N}{k} E_{\text {member }}=\right. \\
& l\left(2 N E_{\text {elec }}+\frac{M^{2}}{2 k \pi} N \varepsilon_{f_{s}}+E_{D A} N+k \varepsilon_{f s} d_{t o B S}^{2}\right)
\end{aligned}
$$

Assuming that the nodes are uniformly distributed, with $\rho(x, y)$ represent the node distribution, it can be shown that:

$$
d_{t o C H}^{2}=\iint\left(x^{2}+y^{2}\right) \rho(x, y) d x d y=\frac{M^{2}}{2 \pi k}
$$

As the average distance from a cluster head to the sink is given by [22]:

$$
d_{t o B S}=\int_{A} \sqrt{x^{2}+y^{2}} \frac{1}{A} d A=0.765 \frac{M}{2}
$$

By differentiating $E_{\text {total }}$ respect to $k$ and equating to zero, the relative optimal number of constructed clusters $k_{\text {opt }}$ can be found as:

$$
k_{\text {opt }}=\sqrt{\frac{N}{2 \pi}} \frac{M}{d_{t o B S}}=\sqrt{\frac{N}{2 \pi}} \frac{2}{0.765}
$$

The optimal probability of a node to become a cluster head $p_{\text {opt }}$ can be computed as follows:

$$
p_{\text {opt }}=\frac{k_{\text {opt }}}{N}=\frac{1}{0.765} \sqrt{\frac{2}{\pi N}}
$$

It is interesting to notice that the optimal probability of a node to become a cluster head is independent of the scale of the sensing field and only related to the number of sensor nodes.

\subsection{Cluster head selection}

The density of a node Den, is set to represent the number of neighbor nodes located within its transmission range. With itself as the center and $R$ as the radius, the density of node $s_{i}$ can be calculated via searching the entire network as Eq. (12), where $d\left(s_{i}, s_{x}\right)$ represents the distance between $s_{i}$ and another node $s_{x}$. 


$$
\operatorname{Den}\left(s_{i}\right)=\text { number of } x, \quad 0<x \leq N \& d\left(s_{i}, s_{x}\right) \leq R
$$

We set parameter $\alpha$ to represent the relative Den of nodes:

$$
\alpha=\frac{\operatorname{Den}\left(s_{i}\right)}{\operatorname{Den}_{\text {avg }}}=\frac{\operatorname{Den}\left(s_{i}\right)}{\sum_{0<i<N} \operatorname{Den}\left(s_{i}\right) / N}
$$

where $\alpha$ is the average density of all nodes. The larger $\alpha$ is, the more neighbors the node has. Namely, it can cover more nodes as a cluster head.

As nodes are heterogeneous in energy capacity, DECHA also select cluster heads according to their energy condition. Not only is the initial energy capacity valued, but also its residual energy after previous rounds of operation.

Here, we have the initial energy of a node noted as $E_{\text {init }}$. The relative initial energy of a node can be represented by parameter $\beta$ as follows:

$$
\beta=\frac{E_{i n i}}{\sum_{0<i<N} E_{i n i}\left(s_{i}\right) / N}
$$

It indicates that nodes with different initial energy can be evaluated respectively, which is more suitable for the multi-level heterogeneous WSNs.

We also assume a maximum value of round $r_{\max }$. In round $r$, the residual energy of each node is noted as $E_{r e s i}$. The average residual energy of nodes can be calculated as

$$
E_{\text {avg }}=\frac{\left(1-r / r_{\max }\right) E_{\text {total }}}{N}
$$

A parameter $\gamma$ stands for its relative residual energy among all nodes.

$$
\gamma=\frac{E_{\text {resi }}}{E_{\text {avg }}}
$$

In our DECHA, besides the density of a node, both its original energy and current residual energy are evaluated as important metrics for $\mathrm{CH}$ selection. We determine the cluster heads according to the election probability of each node following the thoughts of LEACH. With both energy capacity and distribution of $\mathrm{CHs}$ into consideration, the probability is weighted as follows:

$$
p\left(s_{i}\right)=p_{\text {opt }} \cdot \alpha \cdot \beta \cdot \gamma=p_{\text {opt }} \cdot \frac{N \cdot \operatorname{Den}\left(s_{i}\right)}{\sum_{0<i<N} \operatorname{Den}\left(s_{i}\right)} \cdot \frac{N \cdot E_{\text {ini }}}{E_{\text {total }}} \cdot \frac{E_{\text {resi }}}{E_{\text {avg }}}
$$

A node with much energy and good location turns to have a relative larger $p\left(s_{i}\right)$, therefore it has more chance to be a $\mathrm{CH}$. However, as cluster heads are still elected with randomization, the probability cannot fully decide. We regard them as candidate $\mathrm{CHs}$. In the next step, a metric $\eta$ is evaluated. It aims to judge whether the chosen candidate $\mathrm{CH}$ is reasonable.

$$
\eta=\frac{E_{\text {resi }}}{E_{\text {init }}}
$$

From the above formula, we can see $\eta$ represents the proportion of the residual energy and initial energy of a node. In this paper, we pre-determine a threshold number $\eta_{\text {threshold }}$ such as $10 \%$. Once $\eta \leq \eta_{\text {threshold }}$, the cluster head lacks capacity and its $\mathrm{CH}$ role can be given to one of its neighbor randomly. 
A metric denoted as Level is defined and evaluated among the neighbors of a candidate $\mathrm{CH}$. It represents the energy level for data transmission and can adjust the candidate to a more proper $\mathrm{CH}$ in practice.

$$
\operatorname{Level}\left(s_{i}\right)=\frac{E_{\text {resi }}\left(s_{i}\right)}{\sum_{0<x \leq N \& d\left(s_{i}, C H\right) \leq R} \operatorname{Cost}\left(s_{i}\right) / \operatorname{Den}\left(s_{i}\right)}
$$

where $\operatorname{Cost}\left(s_{i}\right)$ denotes the energy consumption for the $\mathrm{CH}$ to communicate with its neighbor within the transmission range; $\sum_{0<x \leq N \& d\left(s_{i}, C H\right) \leq R} \operatorname{Cost}\left(s_{i}\right) / \operatorname{Den}\left(s_{i}\right)$ represents the average energy consumption among its neighbors, namely a standard of average cost of energy in its cluster. The metric aims to find nodes with relative more residual energy and less transmission cost in neighborhood. Nodes with larger Level is set as the actual cluster heads in current round. It is energy-efficient with regard to data transmission.

\subsection{Routing procedure}

After determining all cluster heads, sensor nodes send data to one cluster head directly within one hop. The corresponding cluster head should be determined with the least energy consumption as the transmission cost along the path. According to Eq. (2) in the energy model, distance plays a significant role. We can use the distance between nodes rather than precise information to define the energy cost along the path. Therefore the intra-routing algorithm can be formulated as to find:

$$
\underset{k}{\operatorname{Min}}\left(d\left(s_{i}, \mathrm{CH}_{k}\right)\right), \quad \mathrm{O}<i<N \& s_{i} \neq \mathrm{CH}_{k}
$$

In algorithms as LEACH, cluster heads send data to the base station directly within one hop. There is high chance that it consumes large energy due to the remote location of some cluster head. In our DEGRA, energy efficiency is one of our top concerns. That is we aim at decreasing the energy cost per packet. Here, we perform a multi-hop inter-cluster algorithm.

For an arbitrary cluster head, optimal relay cluster heads are found that can bring the least energy consumption along the path. It is implemented via the greedy forwarding algorithm [23]. Compare it with the direct communication cost to $B S$.

Take 2-hop route as an example, suppose that cluster head $\mathrm{CH}_{i}$ chooses another cluster head $\mathrm{CH}_{j}$ as its relay node. In order to deliver a l-length packet to $\mathrm{BS}$ via $\mathrm{CH}_{j}$, the energy consumed of $\mathrm{CH}_{i}$ is calculated as:

$$
\begin{aligned}
E_{\text {relay }}\left(\mathrm{CH}_{j}\right) & =E_{T x}\left(l, d\left(\mathrm{CH}_{i}, \mathrm{CH}_{j}\right)\right)+E_{R x}(l)+E_{T x}\left(l, d\left(\mathrm{CH}_{j}, \mathrm{BS}\right)\right) \\
& =l\left(E_{\text {elec }}+\varepsilon_{f s} d^{2}\left(\mathrm{CH}_{i}, C H_{j}\right)\right)+l E_{\text {elec }}+\left(l E_{\text {elec }}+\varepsilon_{f s} d^{2}\left(C H_{j}, B S\right)\right) \\
& =3 l E_{\text {elec }}+l \varepsilon_{f s}\left(d^{2}\left(C H_{i}, C H_{j}\right)+d^{2}\left(C H_{j}, B S\right)\right)
\end{aligned}
$$

The direct communication cost to $B S$ is calculated as:

$$
E_{\text {direct }}=l\left(E_{\text {elec }}+\varepsilon_{f s} d^{2}\left(C H_{i}, B S\right)\right)
$$

Compare $d^{2}\left(\mathrm{CH}_{i}, \mathrm{CH}_{j}\right)+d^{2}\left(\mathrm{CH}_{j}, \mathrm{BS}\right)$ and $d^{2}\left(\mathrm{CH}_{i}, \mathrm{BS}\right)$ for simplicity, and the path with a smaller value survives. 


\section{Performance Evaluation}

\subsection{Simulation environment}

We evaluate the performance of the DECHA via simulations in MATLAB. The environment is set up with the parameters listed in Table 1.

Table 1. Network parameters

\begin{tabular}{|c|c|}
\hline Parameter Name & Value \\
\hline Number of the sensor nodes $(N)$ & 100 \\
\hline Length of the packet $(l)$ & $4000 \mathrm{bits}$ \\
\hline Initial energy of normal/advance nodes $\left(E_{\text {init }}\right)$ & $0.5 \sim 1 \mathrm{~J} / 1.5 \sim 2 \mathrm{~J}$ \\
\hline Energy consumption on circuit $\left(E_{\text {elec }}\right)$ & $50 \mathrm{~nJ} / \mathrm{bit}$ \\
\hline Channel parameter in free-space model $\left(\varepsilon_{f s}\right)$ & $10 \mathrm{pJ} / \mathrm{bit} / \mathrm{m}^{2}$ \\
\hline Channel parameter in multi-path model $\left(\varepsilon_{m p}\right)$ & $0.0013 \mathrm{pJ} / \mathrm{bit} / \mathrm{m}^{4}$ \\
\hline Channel parameter for data aggregation $\left(\varepsilon_{D A}\right)$ & $5 \mathrm{pJ} / \mathrm{bit} / \mathrm{signal}$ \\
\hline Pre-determined energy level of cluster heads $\left(\eta_{\text {threshold }}\right)$ & $10 \%$ \\
\hline
\end{tabular}

\subsection{Simulation results}

Figure 2 shows the scenario of a uniform dispersion of 100 sensor nodes in a $500 \times 500 \mathrm{~m}^{2}$ square region. Without loss of generality, here we assume that the base station is located at the coordination of $(250,250)$.

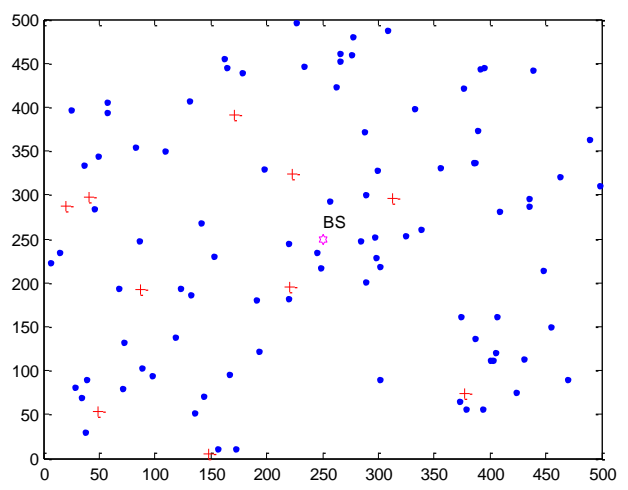

Figure 2. Stimulation network

Let " $\square$ " represent cluster heads of certain round. Figure 3 shows the distribution of cluster heads in LEACH for a certain round. As it adopts randomization with little restraints about the selection procedure, there is high chance that some cluster heads locate relatively close to each other (e.g., the center-right part, where four $\mathrm{CHs}$ locate nearby), which wastes resource; or in some area nodes have to transmit data to remote cluster head (e.g., the center-left part, where none $\mathrm{CH}$ is selected), which costs traffic. Moreover, nodes share same reparability to be a $\mathrm{CH}$, so nodes with less energy may suffer from heavy traffic load as cluster heads and expire rapidly. It is obviously not suitable for heterogeneous network. 


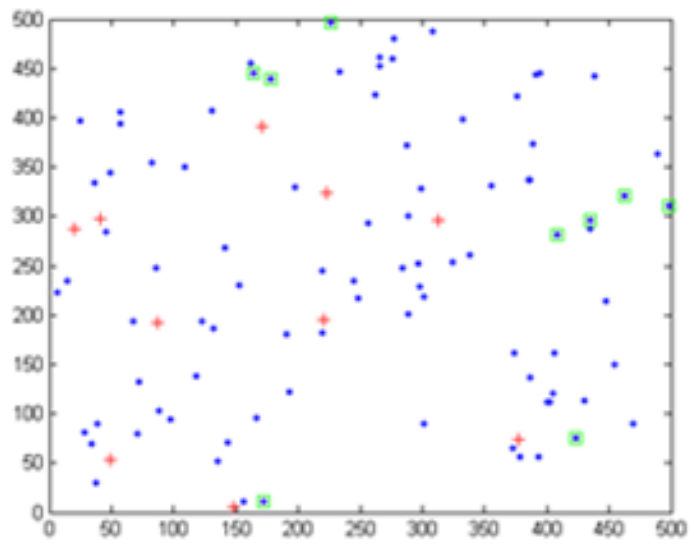

Figure 3. Cluster head distribution of LEACH

In our DECHA, with nodes' feature and distribution under consideration, cluster heads are elected much more properly in the heterogeneous network as is shown in Figure 4. Nodes with relative more energy are more likely to be selected as $\mathrm{CHs}$, which lessens burden of normal nodes. And different from SEP and DEEC, the density metric in DECHA actually promotes that $\mathrm{CHs}$ locate more evenly. It avoids unnecessarily close $\mathrm{CHs}$ and ensures that nodes have its $\mathrm{CH}$ relative nearby. For example, in the upper-right part in Figure 4, none advance nodes exist. For SEP or DEEC which puts priority to nodes' energy, it may have little chance to elect a $\mathrm{CH}$ in such area, and thus nodes have to transmit data to remote $\mathrm{CHs}$ which costs a lot. In our DECHA, however, a node $(385,337)$ is set as a $\mathrm{CH}$ to provide service for the isolated area. It can save energy for the entire network.

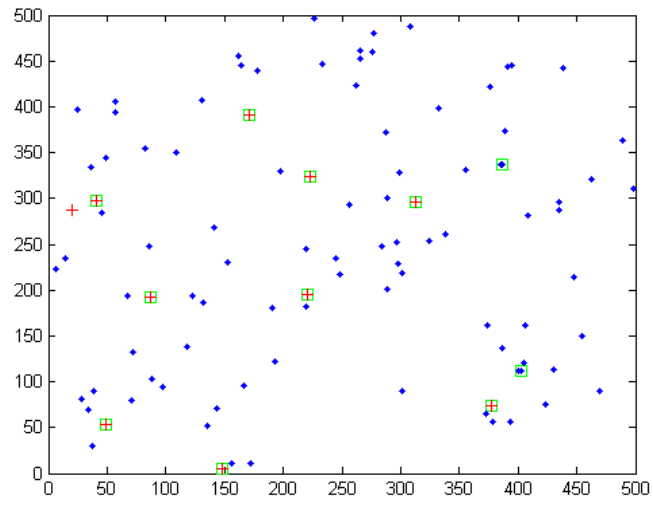

Figure 4. Cluster head selection of DECHA

We compare the network lifetime of LEACH, SEP, DEEC and our DECHA, as is shown in Figure 5 where the network is set as $500 \times 500 \mathrm{~m}^{2}$. For LEACH, all nodes become invalid in $1195^{\text {th }}$ round; for SEP, nodes die out in $2550^{\text {th }}$ round; DEEC has all nodes invalid in $4695^{\text {th }}$ round; DECHA has last node die in $4924^{\text {th }}$ round and shows the best performance as the first node is found in $249^{\text {th }}$ round, which is obviously much later than all three algorithms. It proves a longer stability period of the network. It is mainly due to the dynamic changes of cluster head roles considering nodes' energy and distribution. Moreover the multi-hop intracluster routing algorithm does save energy and enhance the network lifetime to some extent. 


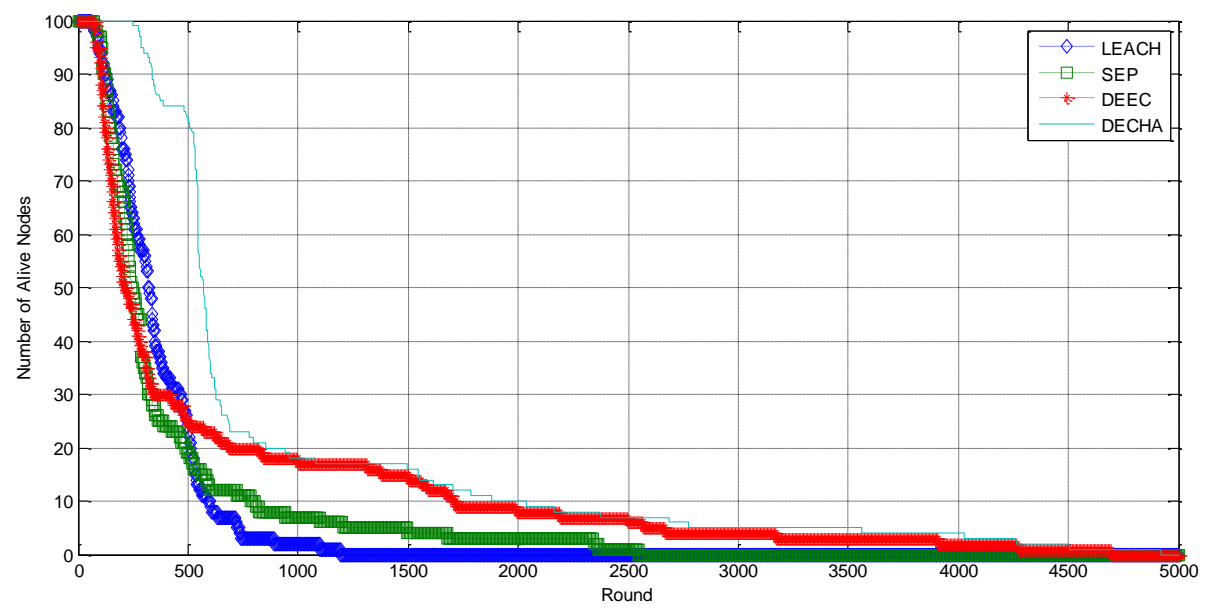

Figure 5. Comparison of network lifetime

We compare the total energy consumption of LEACH, SEP, DEEC and our DECHA, as is shown in Figure 6 where the network is set as $500 \times 500 \mathrm{~m}^{2}$. During 5000 rounds, the energy consumption of LEACH, SEP and DEEC are quite similar. It is because they focus on the selection of cluster heads in each round and fail to consider how to save energy. In comparison, DECHA shows better performance with much less total energy consumption than all of the other algorithms. This is because the clusters are evenly divided and reduce possibility of wasting resources. In addition, the multi-hop intra-cluster method saves much energy as well.

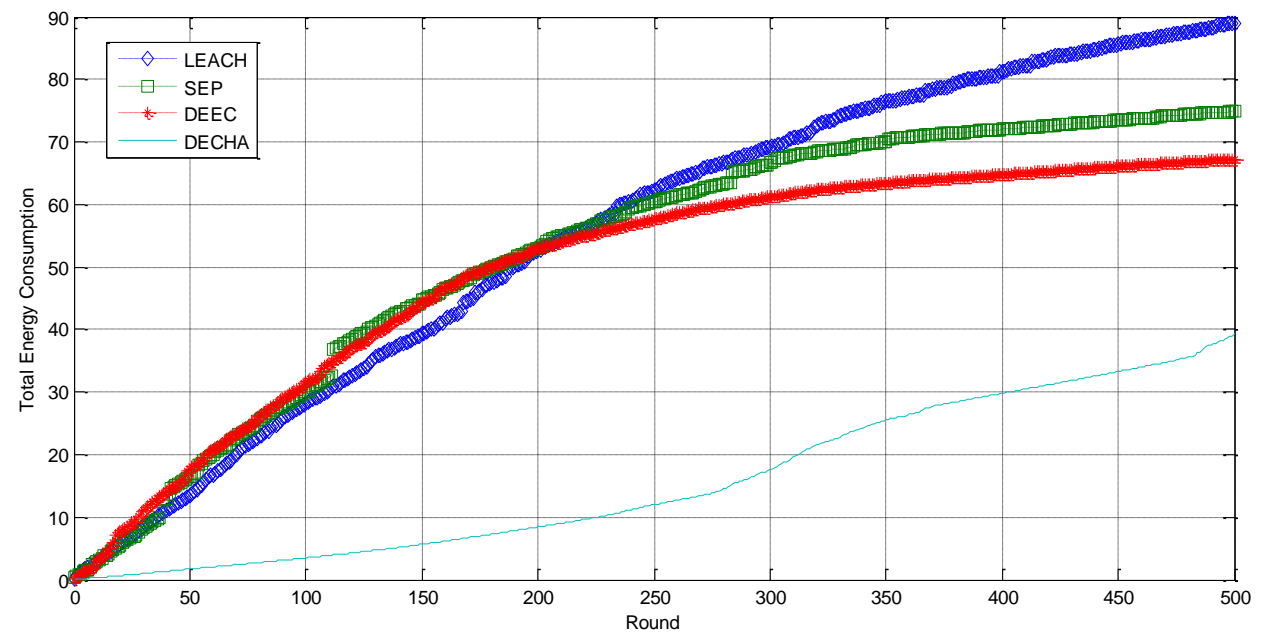

Figure 6. Comparison of total energy consumption

\section{Conclusions}

In this paper, we propose a Density-based Energy-efficient Clustering Heterogeneous Algorithm (DECHA) for WSNs. It is especially suitable for the heterogeneous environment. 
Via considering the position information and energy capacity of nodes, it dynamically changes the probability of nodes to become a candidate cluster head. Metrics as energy level further adjust the $\mathrm{CH}$ selection in a balanced and efficient way. An intra-cluster routing algorithm and a multi-hop inter-cluster routing algorithm are proposed. Optimal probability of $\mathrm{CH}$ is described. Simulations show that the distribution of $\mathrm{CHs}$ is reasonable in DECHA. Both stability period and network lifetime become longer and energy consumption is largely reduced and with comparison to some existed algorithms.

\section{Acknowledgements}

This work was supported by Jiangsu Province Universities Natural Science Research Program (NO.11KJB510010) and Jiangsu Province Research and Innovation Project for College Graduates (NO.CXZZ12_0515). It was also supported by the Industrial Strategic Technology Development Program (10041740) funded by the Ministry of Trade, Industry and Energy (MOTIE Korea), and by the Natural Science Foundation of Jiangsu Province (No. BK2012461). Professor Jeong-Uk Kim is the corresponding author.

\section{References}

[1] I. Akyildiz, W. Su, Y. Sankarasubramaniam and E. Cayirci, "A Survey on Sensor Networks", IEEE Commun. Mag, vol. 8, no. 102, (2002).

[2] E. J. Duarte-Melo and M. Liu, "Analysis of Energy Consumption and Lifetime of Heterogeneous Wireless Sensor Networks", Proceedings of the IEEE Global Communications Conference, Taipei, Taiwan, (2002) November 17- 21.

[3] H. Y. Mark, K. Nandakishore, Singh and Harkirat, "Exploiting Heterogeneity in Sensor Networks", Proceedings of IEEE 24th Annual Joint Conference of the IEEE Computer and Communications Societies, Miami, FL, United States, (2005) March 13-17.

[4] W. Heinzelman, A. Chandrakasan and H. Balakrishnan, "Energy-efficient Routing Protocols for Wireless Microsensor Networks", Proceedings of the 33rd Annual Hawaii International Conference of System Sciences, Maui, Hawaii, (2000) January 4-7.

[5] S. Lindsey and C. S. Raghavendra, "PEGASIS: Power-efficient Gathering in Sensor Information Systems", Parallel and Distributed Systems, vol. 9, no. 924, (2002).

[6] O. Younis and S. Fahmy, "HEED: A Hybrid, Energy-efficient", Distributed Clustering Approach for Ad Hoc Sensor Networks. Mobile Compu, vol. 3, no. 366, (2004).

[7] M. Ye, C. Li, G. Chen and J. Wu, "EECS: An Energy Efficient Cluster Scheme in Wireless Sensor Networks", Proceedings of IEEE International Workshop on Strategies for Energy Efficiency in Ad Hoc and Sensor Networks, Phoenix, Arizona, (2005) April 7-9.

[8] J. M. Kim, S. H. Park and Y. J. Han, "CHEF: Cluster Head Election Mechanism Using Fuzzy Logic in Wireless Sensor Networks", Advanced Communication Technology, vol. 1, no. 654, (2008).

[9] Y. Wang, T. L. X. Yang and D. Zhang, "An Energy Efficient and Balance Hierarchical Unequal Clustering Algorithm for Large Scale Sensor Networks", Information Technology Journal, vol. 8, no. 28, (2009).

[10] B. S. Lee, H. W. Lin and W. Tarng, "A Cluster Allocation and Routing Algorithm Based on Node Density Extending the Lifetime of Wireless Sensor Networks", Proceedings of the 26th International Conference on Information Networking and Applications Workshops (WAINA), Fukuok, Japan, (2012) March 26-29.

[11] M. C. M. Thein and T. Thein, "An Energy Efficient Cluster-head Selection for Wireless Sensor Networks", Proceedings of International Conference on Intelligent Systems, Modelling and Simulation (ISMS), Liverpool, United Kingdom, (2010) January 27-29.

[12] N. Xu, A. P. Huang, T. W. Hou and H. H. Chen, "Coverage and Connectivity Guaranteed Topology Control Algorithm for Cluster-based Wireless Sensor Networks", Wireless Communications and Mobile Computing, vol. 12, no. 23, (2012).

[13] J. F. Qiao, S. Y. Liu and X. Y. Cao, "Density-based Clustering Protocol for Wireless Sensor Networks", Computer Science, vol. 12, no. 46, (2009).

[14] G. Smaragdakis, I. Matta and A. Bestavros, "SEP: A Stable Election Protocol for Clustered Heterogeneous Wireless Sensor Networks", Proceedings of Second International Workshop on Sensor and Actor Network Protocols and Applications, Boston, Massachusetts, USA, (2004) August 22. 
[15] L. Qing, Q. Zhu and M. Wang, "Design of a Distributed Energy-efficient Clustering Algorithm for Heterogeneous Wireless Sensor Networks", Computer Communications, vol. 29, no. 2230, (2006).

[16] X. L. Xu and J. N. Qiu, Energy Efficient Cluster-Head Selection for Heterogeneous Wireless Sensor Networks", Chinese Journal of Sensors and Actuators, vol. 22, no. 3, (2009).

[17] D. Kumar, T. C. Aseri and R.B. Patel", EEHC: Energy Efficient Heterogeneous Clustered Scheme for Wireless Sensor Networks", Computer Communications, vol. 32, no. 662, (2009).

[18] J. Y. Yin, "Research on Clustering Algorithm in Heterogeneous Wireless Sensor Networks", MS thesis, Harbin Institute of Technology, (2011).

[19] N. Javaid, T. N. Qureshi, A. H. Khan, A. Iqbal, E. Akhtar and M. Ishfaq, "EDDEEC: Enhanced Developed Distributed Energy-Efficient Clustering for Heterogeneous Wireless Sensor Networks", Procedia Computer Science, vol. 19, no. 914, (2013).

[20] R. Tandon and S. Nandi, "CRP: Cluster Head Re-election Protocol for Heterogeneous Wireless Sensor Networks", Proceedings of Fifth International Conference on Communication Systems and Networks (COMSNETS), Bangalore, India, (2013) January 7-10.

[21] T. Rappaport, "Wireless Communications: Principles \& Practice”, Englewood Cliffs, Prentice-Hall, (1996).

[22] S. Bandyopadhyay and E. J. Coyle, "Minimizing Communication Costs in Hierarchically-clustered Networks of Wireless Sensors", Comput. Networks, vol. 44, no. 1, (2004).

[23] B. Karp and H. T. Kung, "GPSR: Greedy Perimeter Stateless Routing for Wireless Networks", Proceedings of the 6th Annual International Conference on Mobile Computing and Networking, Boston, Massachusetts, (2000) August 6-11.

\section{Authors}



\section{Zhanyang Xu}

Zhanyang Xu obtained his Masters in Computer Engineering from the School of Computer Science and Technology, China University of Mining and Technology (CUMT) in 2004. He graduated as the top student in the Nanjing University of Posts and Telecommunications (NJUPT) where would obtain his PhD in 2012. At the same time, he is serving as a full time faculty in the School of Computer \& Software, Nanjing University of Information Science \& Technology (NUIST). His research interest includes wireless communication network, wireless sensor networks and Internet of things.

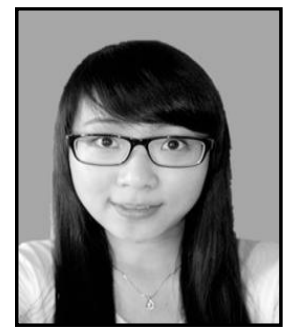

\section{Yue Yin}

Yue Yin received the Bachelor degree in Applied Computing from Nanjing University of Information and Science Technology in 2011. She is currently pursuing a Master degree in Technology of Computer Application in the former institution. Her research interests include wireless sensor networks and Internet of things, specifically in fields of algorithm design and improvement, performance evaluation, game theory and data replication. 


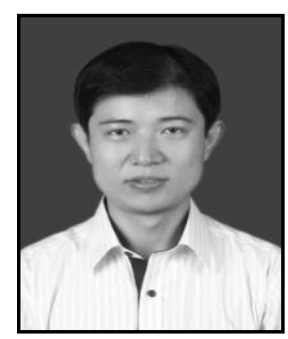

\section{Jin Wang}

Jin Wang received his B.S. and M.S. degree in the Electronical Engineering from Nanjing University of Posts and Telecommunications, China in 2002 and 2005, respectively. He received Ph.D. degree from the Computer Engineering Department of Kyung Hee University Korea in 2010. Now, he is a professor in the Computer and Software Institute, Nanjing University of Information Science and Technology. He has published more than 120 journal and conference papers. His main research interests include routing protocol and algorithm design, performance evaluation and optimization for wireless sensor networks.

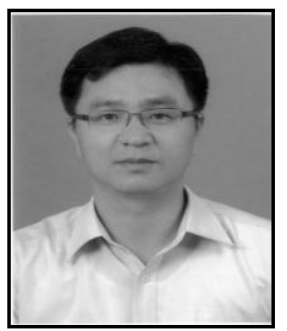

\section{Jeong-Uk Kim}

He obtained his B.S. degree in Control and Instrumentation Engineering from Seoul National University in 1987, M.S. and Ph.D. degrees in Electrical Engineering from Korea Advanced Institute of Science and Technology in 1989, and 1993, respectively. He is a professor in Sangmyung University in Seoul. His research interests include smart grid demand response, building automation system, and renewable energy. 
International Journal of Control and Automation Vol.7, No.2 (2014) 